\title{
Disturbance response decoupling and achievable performance with application to vehicle active suspension
}

\author{
FU-CHENG WANG ${ }^{\dagger}$ and MALCOLM C. SMITH ${ }^{\dagger *}$
}

This paper derives a structural condition on the controller for a given (stable) plant which guarantees that some prespecified closed-loop transfer function is the same as in the open loop. We also present conditions to test whether the achievable dynamic response of other transmission paths remains effectively the same if the controller is so restricted. The results are applied to simple quarter- and half-car vehicle models, and illustrated numerically for a double-wishbone halfcar model.

\section{Introduction}

This paper continues the work of Smith and Wang (2002) to derive a new condition on the controller structure for a given (stable) plant which guarantees that some pre-specified closed-loop transfer function is fixed. The controller consists of two blocks in series, the first of which is a left annihilator of a certain open-loop plant transfer function and the second is arbitrary. As such, the condition takes a simpler form than that of Smith and Wang (2002), by assuming that the plant is open-loop stable and the number of outputs to be left invariant is no smaller than the number of actuators and that this transmission path has full normalrank.

The motivation for this problem comes from vehicle active suspension control (Thompson 1971, Pitcher et al. 1977, Hrovat and Hubbard 1981, Wright and Williams 1984, Sharp and Hassan 1986, Smith 1995) where there is a need to insulate the vehicle body from both road irregularities and load disturbances (e.g. inertial loads induced by braking and cornering). It is frequently the case that the hardware structure ensures that some disturbance paths are satisfactory in the open-loop, e.g. the response to road disturbances (Pitcher et al. 1977, Williams et al. 1993, Williams and Best 1994). In Smith and Wang (2002) and Wang and Smith (2001) the authors introduced the idea of parametrizing all stabilizing controllers to leave some transmission path invariant and derived some general conditions to achieve this.

This paper also considers a new issue related to our disturbance response decoupling problem, namely whether the achievable performance in other transmission paths is reduced when the controller is restricted in form. We will derive conditions to determine whether the achievable performance remains effectively the same.

Received 7 November 2001. Revised 10 May 2002.

* Author for correspondence. e-mail: mcs@eng.cam.ac.uk

$\dagger$ Department of Engineering, University of Cambridge, Cambridge CB2 1PZ UK.
The paper is outlined as follows. Section 2 derives the main results of the paper. Theorem 1 sets up the controller structure as a left annihilator for a given plant. Theorem 2 derives the conditions under which the achievable performance of other transmission paths remains effectively unchanged. Section 3 applies the results of $\S 2$ to the standard quarter- and half-car models employing a 'Sharp' actuator with various choices of measured variables. Section 4 presents a numerical example which derives the required controller structure for a half-car double-wishbone model. The multi-body simulation package AutoSim was employed to derive the linearized model and to perform the nonlinear simulations.

\section{Controller structure for disturbance response decoupling}

We consider the LFT (linear fractional transformation) model in figure 1, where the Laplace transfer function of the generalised plant $P$ is partitioned as

$$
P=\left[\begin{array}{ll}
P_{11} & P_{12} \\
P_{21} & P_{22}
\end{array}\right]
$$

and further partitioned conformably with the disturbance signals as

$$
\left.\left[\begin{array}{c}
\hat{z}_{1} \\
\hat{z}_{2}
\end{array}\right]=\left[\begin{array}{cc}
{\left[\begin{array}{ll}
P_{11,11} & P_{11,12} \\
P_{11,21} & P_{11,22}
\end{array}\right]} & {\left[\begin{array}{c}
P_{12,1} \\
P_{12,2}
\end{array}\right]} \\
{\left[\begin{array}{ll}
P_{21,1} & P_{21,2}
\end{array}\right]} & P_{22}
\end{array}\right]\left[\begin{array}{c}
{\left[\hat{w}_{1}\right.} \\
\hat{w}_{2}
\end{array}\right]\right]
$$

where $w_{1} \in \mathbb{R}^{m_{1}}, w_{2} \in \mathbb{R}^{m_{2}}, u \in \mathbb{R}^{m_{3}}, z_{1} \in \mathbb{R}^{p_{1}}, z_{2} \in \mathbb{R}^{p_{2}}$, $y \in \mathbb{R}^{p_{3}}$ at any time instant and $\hat{u}$ denotes the Laplace transform of $u(t)$, etc.

We consider the problem of parametrizing all stabilizing controllers which leave $T_{\hat{w}_{1} \rightarrow \hat{z}_{1}}$ (the transfer function from $\hat{w}_{1}$ to $\hat{z}_{1}$ ) the same as in the open-loop. To make sense, this problem requires that $P$ is stable, which will indeed be satisfied in our application. In this 


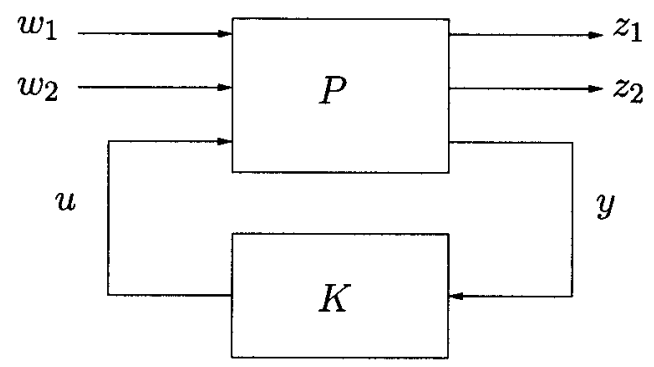

Figure 1. Generalized model in LFT form.

case, the set of all stabilizing controllers can be parametrized by

$$
K=-\left(I-Q P_{22}\right)^{-1} Q
$$

for $Q \in \mathbb{R} \mathbb{H}_{\infty}^{m_{3} \times p_{3}}$. The closed loop transfer function in figure 1 can be expressed as

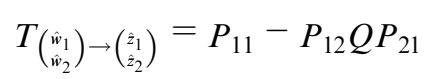

Thus the problem reduces to parametrizing the subset of all stabilizing controllers which leave $T_{\hat{w}_{1} \rightarrow \hat{z}_{1}}=(P)_{11,11}$. To facilitate the derivation, we denote the normalrank of $P_{12,1}$ and $P_{21,1}$ as $r_{2}$ and $r_{3}$ respectively. In Smith and Wang (2002), the authors developed a general theorem for the above problem. Now we will explore the problem from a different angle in the following theorem.

Theorem 1: Let $P$ be (open-loop) stable. Consider the controller structure of figure 2 with $P$ given by (1). Then

(i) $T_{\hat{w}_{1} \rightarrow \hat{z}_{1}}=P_{11,11}$ if $\tilde{U}_{2}$ is a left annihilator of $P_{21,1}$.

(ii) If $P_{12,1}$ has full column normalrank and $T_{\hat{w}_{1} \rightarrow \hat{z}_{1}}=P_{11,11}$, then $K_{1} U_{2}$ is a left annihilator of $P_{21,1}$.

(iii) Assume $m_{3}=r_{2}$ and let $\tilde{U}_{2} \in \mathbb{R} \mathbb{H}_{\infty}^{\left(p_{3}-r_{3}\right) \times p_{3}}$ be a part of a unimodular matrix and be a left annihilator of $P_{21,1}$. Then $K=K_{1} U_{2}$, where $K_{1}=$

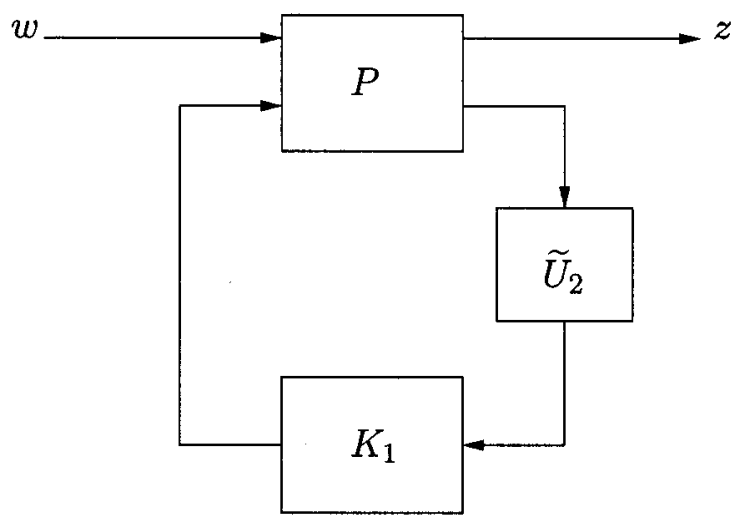

Figure 2. Controller structure as a left annihilator.
$-\left(I-Q_{1} \tilde{U}_{2} P_{22}\right)^{-1} Q_{1} \quad$ and $\quad Q_{1} \in \mathbb{R} \mathbb{H}_{\infty}^{m_{3}} \times\left(p_{3}-r_{3}\right)$, parametrizes all stabilizing controllers of $P$ for which $T_{\hat{w}_{1} \rightarrow \hat{z}_{1}}=P_{11,11}$.

\section{Proof:}

(i) The closed-loop response of the system is $T_{\tilde{w}_{\hat{w}} \rightarrow \hat{z}}=P_{11}+P_{12}\left(I-K_{1} U_{2} P_{22}\right)^{-1} K_{1} \underset{\sim}{U_{2}} P_{21} . \quad$ If $\widetilde{U}_{2}$ is a left annihilator of $P_{21,1}$, i.e. $\widetilde{U}_{2} P_{21,1}=0$, then

$$
\begin{aligned}
T_{\hat{w}_{1} \rightarrow \hat{z}_{1}} & =P_{11,11}+P_{12,1}\left(I-K_{1} \tilde{U}_{2} P_{22}\right)^{-1} K_{1} \tilde{U}_{2} P_{21,1} \\
& =P_{11,11}
\end{aligned}
$$

which is invariant of the feedback control.

(ii) If $P_{12,1}$ has full column normalrank and (4) holds for some controller $K_{1}$, then $K_{1} U_{2} P_{21,1}=0$. That is $K_{1} U_{2}$ is a left annihilator of $P_{21,1}$.

(iii) Suppose $\tilde{U}_{1^{1}} \in \mathbb{R} \mathbb{H}_{\sim}^{r_{3}}{ }^{\times p_{3}}$ is a completion of $\tilde{U}_{2}$ such that $\widetilde{U}=\left(\widetilde{U_{1}^{\prime}}, \widetilde{U_{2}^{\prime}}\right)^{\prime}$ is unimodular, and $U^{-1}=U=\left(U_{1}, U_{2}\right)$. Since $U_{2}$ is a left annihilator of $P_{21,1}$, we can write

$$
\widetilde{U} P_{21,1}=\left[\begin{array}{c}
\tilde{U}_{1} \\
\widetilde{U}_{2}
\end{array}\right] P_{21,1}=\left[\begin{array}{l}
F \\
0
\end{array}\right]
$$

for some $F \in \mathbb{R} \Re_{\infty}^{r_{3} \times m_{1}}$, which gives

$$
P_{21,1}=\left[\begin{array}{ll}
U_{1}, & U_{2}
\end{array}\right]\left[\begin{array}{c}
F \\
0
\end{array}\right]=U_{1} F
$$

where $U_{1} \in \mathbb{R} \mathbb{H}_{\infty}^{p_{3} \times r_{3}}$. Note that $F$ has normalrank $r_{3}$ because $P_{21,1}$ has normalrank $r_{3}$ and the multiplication of a unimodular matrix to obtain $F$ does not change the normalrank. Hence, $F$ has full row normalrank.

A stabilizing controller in the form (2) leaves $T_{\hat{w}_{1} \rightarrow \hat{z}_{1}}=P_{11,11}$ if and only if $\left(P_{12} Q P_{21}\right)_{1,1}=P_{12,1} Q U_{1} F=0$. This is equivalent to $Q U_{1}=0$ since $P_{12,1}$ (resp. $F$ ) has full column (resp. row) normalrank. We now show that this requires $Q=Q_{1} U_{2}$ for some $Q_{1}$. Clearly

$$
Q\left(U_{1}, U_{2}\right)=\left(0_{r_{2} \times r_{3}}, Q_{1}\right)
$$

for some $Q_{1} \in \mathbb{R} \mathbb{H} \mathbb{H}_{\infty}^{r_{2} \times\left(p_{3}-r_{3}\right)}$. This gives

$$
Q=\left(0, Q_{1}\right) U^{-1}=Q_{1} \tilde{U}_{2}
$$

Conversely, if $Q=Q_{1} \tilde{U}_{2}$ holds for some $Q_{1} \in \mathbb{R} \mathbb{H}_{\infty}^{r_{0}} \times{ }_{\left(p_{3}-r_{3}\right)}$, then we have $Q=$ $\left(0, Q_{1}\right) U^{-1}$ which again implies $Q U_{1}=0$. Hence $T_{\hat{w}_{1} \rightarrow \hat{z}_{1}}=P_{11,11}$

Remark 1: The idea of Theorem 1(iii) is to 'shrink' the parametrization set of all stabilizing controllers from $Q \in \mathbb{R} \mathbb{R}_{\infty}^{m_{3} \times p_{3}}$ to $Q$ in the form of (6). The form 
of the result in Theorem 1 is more explicit than Smith and Wang (2002), though less general, and emphasizes the left annihilator property. It is implicit in the proof of Theorem 1(iii), and explicit in Smith and Wang (2002), that a left annihilator of $P_{21,1}$ can be found by constructing a 'left normalrank factorization' (Smith and Wang 2002) $P_{21,1}=U_{1} F$ where $F$ has full row normalrank and $U_{1}$ is part of a unimodular matrix over $\mathbb{R H}_{\infty}$ (see Vidyasagar 1985). It is shown constructively in Smith and Wang (2002) that any matrix with elements in $\mathbb{R H}_{\infty}$ has a left normalrank factorization.

A question which was not addressed in Smith and Wang (2002) is whether the parametrization of Theorem 1 introduces performance limitations on other transmission paths. We will now give a result which allows us to test if the achievable performance is significantly reduced. To do this, we will work directly with the $Q$-parameters in the Youla parametrization. A matrix is said to be proper (resp. strictly proper) if all its elements are proper (resp. strictly proper).

Theorem 2: Given two sets of proper, stable transfer matrices, $A=\left\{G_{1} Q H_{1}: Q\right.$ is a matrix with elements in $\left.\mathbb{R} \mathbb{H}_{\infty}\right\}$ and $B=\left\{G_{2} Q H_{2}: Q\right.$ is a matrix with elements in $\left.\mathbb{R} \mathbb{H}_{\infty}\right\}$, where $G_{2} I=G_{1}$ for some $I$ and $K H_{2}=H_{1}$ for some $\boldsymbol{K}$ :

(i) Suppose $G_{1} Q H_{1}$ is strictly proper in $\mathbb{R H} \mathbb{H}_{\infty}$ for any $Q$ which has elements in $\mathbb{R}_{\infty}, L Q K(1 / s \tau+1)^{r}$ is a matrix with elements in $\mathbb{R} \mathbb{H}_{\infty}$ for some $r>0$ and any $\tau>0$, then for any $R_{1} \in A$ we can find a $R_{2} \in B$ such that $\left\|R_{1}-R_{2}\right\|_{\infty}<\epsilon$ for any $\epsilon>0$.

(ii) If $L Q K$ is a proper matrix for any $Q$ which has elements in $\mathbb{R} \mathbb{H}_{\infty}$, then for any $R_{1} \in A$ we can find a $R_{2} \in B$ such that $R_{1}-R_{2}=0$.

\section{Proof:}

(i) For any $R_{1} \in A$, we take the following candidate for $R_{2}$

$$
R_{2}=R_{1}\left(\frac{1}{s \tau+1}\right)^{r}
$$

for some $\tau>0$. Note that $R_{2} \in B$ since by definition $R_{1}=G_{1} Q H_{1}$ for some $Q$ which has elements $\mathbb{R} \mathbb{H}_{\infty}$ and so

$$
R_{2}=G_{2}\left(L Q K(1 /(s \tau+1))^{r}\right) H_{2}
$$

where

$$
\left(\operatorname{LQK}(1 /(s \tau+1))^{r}\right) H_{2}
$$

has elements in $\mathbb{R} \mathbb{H}_{\infty}$. Hence

$$
\left\|R_{1}-R_{2}\right\|_{\infty}=\left\|R_{1}\left(1-\left(\frac{1}{s \tau+1}\right)^{r}\right)\right\|_{\infty}
$$

We now wish to show that the right-hand side of (7) can be made arbitrary small by taking $\tau$ sufficiently small. Firstly, since $R_{1}$ is strictly proper, given any $\epsilon>0$ we can find an $\omega_{0}(\epsilon)$ such that $\bar{\sigma}\left(R_{1}(j \omega)\right)<\epsilon / 2$ for $\omega>\omega_{0}$, and

$$
\begin{gathered}
\bar{\sigma}_{\omega>\omega_{0}}\left(R_{1}(j \omega)\left(1-\left(\frac{1}{j \omega \tau+1}\right)^{r}\right)\right) \\
\leq 2 \bar{\sigma}_{\omega>\omega_{0}}\left(R_{1}(j \omega)\right)<\epsilon
\end{gathered}
$$

Secondly, for $\omega \in\left[0, \omega_{0}\right], \quad(1-1 /(j \omega \tau+1))^{r}$ can be made as small as we like by choosing a sufficiently small $\tau$. Hence we can choose $\tau$ sufficiently small so that $\bar{\sigma}_{\omega \in\left[0, \omega_{0}\right]}\left(R_{1}\left(1-(1 / j \omega \tau+1)^{r}\right)\right)<\epsilon$ for any $\epsilon>0$. Combining these together, we can ensure that $\bar{\sigma}\left(R_{1}\left(1-1 /(j \omega \tau+1)^{r}\right)\right)<\epsilon$ for all $\omega \geq 0$, and therefore, $\left\|\left(R_{1}-R_{2}\right)(j \omega)\right\|_{\infty}<\epsilon$ by choosing $\tau$ sufficiently small.

(ii) If $L Q K$ is a proper matrix for any $Q$ which has elements in $\mathbb{R} \mathbb{H}_{\infty}$, then for any $R_{1} \in A$ we can set $R_{2}=R_{1}$ since $R_{1}=G_{1} Q H_{1}=G_{2}(L Q K) H_{2} \in B$ because $I Q K$ has elements in $\mathbb{R} \mathbb{W}_{\infty}$.

Corollary 1: Given two sets of scalar, strictly proper transfer functions, $A=\left\{G_{1} Q: Q \in \mathbb{R}_{\infty}\right\} \quad$ and $B=\left\{G_{2} Q: Q \in \mathbb{R} \mathbb{H}_{\infty}\right\}$, where $G_{1}$ is strictly proper in $\mathbb{R} \mathbb{H}_{\infty}$ and $G_{2}=G_{1} \times\left(n_{1} / m_{1}\right)$ in which $m_{1}, n_{1}$ are Hurwitz polynomials with $\operatorname{deg}\left(n_{1}\right)<\operatorname{deg}\left(m_{1}\right)$, for any $R_{1} \in A$ we can find a $R_{2} \in B$ such that $\left\|R_{1}-R_{2}\right\|_{\infty}<\epsilon$ for any $\epsilon>0$. If $\operatorname{deg}\left(n_{1}\right)=\operatorname{deg}\left(m_{1}\right)$ then we can choose $R_{2}=R_{1}$.

\section{Application to vehicle models}

In this section, we will apply the disturbance response decoupling theorems to quarter- and half-car models.

\subsection{The quarter-car model}

We begin with the quarter-car model of figure 3 where the sprung and unsprung masses are $m_{s}$ and $m_{u}$ and the tyre is modelled as a linear spring with constant $k_{t}$. The suspension consists of a passive damper of constant $c_{s}$ in parallel with a series combination of an actuator $A$ and a spring of constant $k_{s}$ (sometimes referred to as a 'Sharp' actuator (Wright and Williams 1989)). Following Sharp and Hassan (1987) the actuator is modelled so that the relative displacement across the actuator will be a low-pass filtered version of the actuator's command signal, i.e.

$$
\hat{z}_{s}-\hat{z}_{a}=\gamma(s) \hat{u}=\frac{\omega_{n}^{2}}{s^{2}+2 \delta \omega_{n} s+\omega_{n}^{2}} \hat{u}
$$

The external disturbances are taken to be a load $F_{s}$ and a road displacement $z_{r}$. The dynamic equations of the 


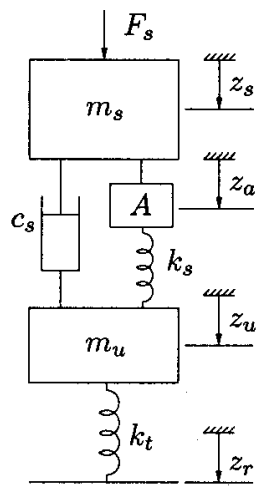

Figure 3. The quarter-car model.

model are given by

$$
\begin{aligned}
& m_{s} \ddot{z}_{s}=F_{s}-u_{p} \\
& m_{u} \ddot{z}_{u}=u_{p}+F_{r}
\end{aligned}
$$

where

$$
\begin{aligned}
& u_{p}=c_{s}\left(\dot{z}_{s}-\dot{z}_{u}\right)+k_{s}\left(z_{a}-z_{u}\right) \\
& F_{r}=k_{t}\left(z_{r}-z_{u}\right)
\end{aligned}
$$

We assume that $k_{s}$ and $c_{s}$ are chosen to give satisfactory responses for the transmission path from $z_{r}$ to $z_{s}$ and $z_{u}$. We therefore seek to parametrize all controllers which leave this transmission path the same as in the open-loop.

3.1.1. Two measurement case. We assume that the measurements $\ddot{z}_{s}$ and $z_{s}-z_{u}$ are available. We now write the system in the form of figure 1 with $z_{1}=$ $\left[z_{s}, z_{u}\right]^{\prime}, w_{1}=z_{r}, w_{2}=F_{s}, y=\left[\ddot{z}_{s}, z_{s}-z_{u}\right]^{\prime}, u$ equals the actuator command signal as in (8) and $z_{2}$ omitted. The corresponding dimensions are $m_{1}=m_{2}=m_{3}=1$, $p_{1}=2, p_{2}=0, p_{3}=2$. We find that

$$
P_{21}=\frac{1}{d(s)}\left[\begin{array}{cc}
k_{t}\left(c_{s} s+k_{s}\right) s^{2} & \left(m_{u} s^{2}+c_{s} s+k_{s}+k_{t}\right) s^{2} \\
-k_{t}\left(m_{s} s^{2}\right) & m_{u} s^{2}+k_{t}
\end{array}\right]
$$

where

$$
\begin{aligned}
d(s)= & \left(m_{s} m_{u}\right) s^{4}+c_{s}\left(m_{s}+m_{u}\right) s^{3}+\left(m_{s}\left(k_{s}+k_{t}\right)\right. \\
& \left.+m_{u} k_{s}\right) s^{2}+c_{s} k_{t} s+k_{s} k_{t}
\end{aligned}
$$

As expected, all roots of $d(s)$ are in the left-half plane, which can be confirmed by the Routh-Hurwitz Criterion. It can be checked that $r_{2}=r_{3}=1$. Following Remark 1 we can construct a left annihilator $U_{2} \in \mathbb{R} \mathbb{H}_{\infty}^{(}\left(p_{3}-r_{3}\right) \times p_{3}$ of $P_{21,1}$

$$
\tilde{U}_{2}=\left[\begin{array}{ll}
\frac{m_{s}}{c_{s} s+k_{s}} & 1
\end{array}\right]
$$

which satisfies all the conditions of Theorem 1(iii).
We now discuss the achievable performance after disturbance response decoupling. The set of all stabilizing controllers is parametrized by (2) with $Q=\left[Q_{\alpha}, Q_{\beta}\right]$, and we can check that the load responses are given by

$$
\left[\begin{array}{c}
\hat{z}_{s} \\
\hat{z}_{u}
\end{array}\right]=\left(P_{11,12}-L_{1}(s) \frac{1}{d(s)}\left[\begin{array}{c}
k_{s}\left(m_{u} s^{2}+k_{t}\right) \\
-k_{s} m_{s} s^{2}
\end{array}\right]\right) \hat{F}_{s}
$$

where

$$
\begin{aligned}
L_{1}(s)= & \gamma(s)\left(\left(m_{u} s^{2}+c_{s} s+k_{s}+k_{t}\right) s^{2} Q_{\alpha}\right. \\
& \left.+\left(m_{u} s^{2}+k_{t}\right) Q_{\beta}\right) / d(s)
\end{aligned}
$$

and $d(s)$ is given in (13). After disturbance response decoupling with the controller structure $U_{2}$ given in (14), we can check that the load responses are given by

$$
\left[\begin{array}{c}
\hat{z}_{s} \\
\hat{z}_{u}
\end{array}\right]=\left(P_{11,12}-L_{2}(s) \frac{1}{d(s)}\left[\begin{array}{c}
k_{s}\left(m_{u} s^{2}+k_{t}\right) \\
-k_{s} m_{s} s^{2}
\end{array}\right]\right) \hat{F}_{s}
$$

where $L_{2}(s)=\gamma(s) Q_{1} /\left(c_{s} s+k_{s}\right)$. To compare the achievable performance, set

$$
\begin{aligned}
Q_{1}= & \left(\left(m_{u} s^{2}+c_{s} s+k_{s}+k_{t}\right) s^{2} Q_{\alpha}\right. \\
& \left.+\left(m_{u} s^{2}+k_{t}\right) Q_{\beta}\right) / d(s)
\end{aligned}
$$

and note that $L_{2}=L_{1} /\left(c_{s} s+k_{s}\right)$. Since $L_{1}(s)$ is strictly proper, it follows from Corollary 1 that $L_{2}(s)$ can approximate $L_{1}(s)$ to an error of $\epsilon$ in the $H_{\infty}$ norm. Hence the freedom in the achievable load responses after disturbance response decoupling is effectively unchanged.

3.1.2. Three measurement case. We continue to illustrate our theory by considering the quarter-car model with the additional measurement $\ddot{z}_{u}$. We now write the system in the form of figure 1 with $y=\left[\ddot{z}_{s}, z_{s}-z_{u}, \ddot{z}_{u}\right]^{\prime}$ and all other variables the same as in the two-measurement case. We find that

$$
P_{21}=\frac{1}{d(s)}\left[\begin{array}{cc}
k_{t}\left(c_{s} s+k_{s}\right) s^{2} & \left(m_{u} s^{2}+c_{s} s+k_{s}+k_{t}\right) s^{2} \\
-k_{t}\left(m_{s} s^{2}\right) & \left(m_{u} s^{2}+k_{t}\right) \\
k_{t}\left(m_{s} s^{2}+c_{s} s+k_{s}\right) s^{2} & \left(c_{s} s+k_{s}\right) s^{2}
\end{array}\right]
$$

where $d(s)$ is given by (13). It can be checked that $p_{3}=3, r_{2}=r_{3}=1$. Following Remark 1 we can construct a left annihilator $U_{2} \in \mathbb{R}_{\mathbb{R}}^{\left(p_{3}-r_{3}\right) \times p_{3}}$ of $P_{21,1}$

$$
\tilde{U}_{2}=\left[\begin{array}{ccc}
1 & 0 & \frac{-\left(c_{s} s+k_{s}\right)}{m_{s} s^{2}+c_{s} s+k_{s}} \\
0 & 1 & \frac{m_{s}}{m_{s} s^{2}+c_{s} s+k_{s}}
\end{array}\right]
$$

which satisfies all the conditions of Theorem 1(iii).

To discuss the achievable performance, the set of all stabilizing controllers is parametrized by (2) with 
$Q=\left[Q_{\gamma}, Q_{\delta}, Q_{\zeta}\right]$, and the load responses are then given by

$$
\left[\begin{array}{c}
\hat{z}_{s} \\
\hat{z}_{u}
\end{array}\right]=\left(P_{11,12}-L_{3}(s) \frac{1}{d(s)}\left[\begin{array}{c}
k_{s}\left(m_{u} s^{2}+k_{t}\right) \\
-k_{s} m_{s} s^{2}
\end{array}\right]\right) \hat{F}_{s}
$$

where

$$
\begin{aligned}
L_{3}(s)= & \gamma(s)\left(\left(m_{u} s^{2}+c_{s} s+k_{s}+k_{t}\right) s^{2} Q_{\gamma}+\left(m_{u} s^{2}+k_{t}\right) Q_{\delta}\right. \\
& \left.+\left(c_{s} s+k_{s}\right) s^{2} Q_{\zeta}\right) / d(s) .
\end{aligned}
$$

We observe (as in Smith 1995, Theorem 7) that any achievable performance with the three measurements can also be achieved by two measurement $\left[\ddot{z}_{s}, z_{s}-z_{u}\right]^{\prime}$ in that for any $L_{3}$ we can achieve a $L_{1}=L_{3}$ by setting $Q_{\alpha}=Q_{\gamma}+Q_{\zeta}\left(c_{s} s+k_{s}\right) /\left(m_{u} s^{2}+c_{s} s+k_{s}+k_{t}\right)$ and $Q_{\beta}=Q_{\delta}$ in $L_{1}(s)$. After disturbance response decoupling with the controller structure $U_{2}$ given in (17), and with the parameter $Q_{1}=\left[Q_{1,1}, Q_{1,2}\right]$, the load responses are given by

$$
\left[\begin{array}{c}
\hat{z}_{s} \\
\hat{z}_{u}
\end{array}\right]=\left(P_{11,12}-L_{4}(s) \frac{1}{d(s)}\left[\begin{array}{c}
k_{s}\left(m_{u} s^{2}+k_{t}\right) \\
-k_{s} m_{s} s^{2}
\end{array}\right]\right) \hat{F}_{s}
$$

where

$$
L_{4}(s)=\gamma(s)\left(s^{2} Q_{1,1}+Q_{1,2}\right) /\left(m_{s} s^{2}+c_{s} s+k_{s}\right)
$$

Therefore, for any $L_{3}$ we can choose $Q_{1,1}, Q_{1,2}$ as follows

$$
\begin{aligned}
Q_{1,1}= & \frac{\left(m_{u} s^{2}+c_{s} s+k_{s}+k_{t}\right)\left(m_{s} s^{2}+c_{s}+k_{s}\right)}{d(s)} \\
& \times\left(Q_{\gamma}+\frac{c_{s} s+k_{s}}{m_{u} s^{2}+c_{s} s+k_{s}+k_{t}} Q_{\zeta}\right) \\
Q_{1,2}= & \frac{\left(m_{u} s^{2}+k_{t}\right)\left(m_{s} s^{2}+c_{s} s+k_{s}\right)}{d(s)} Q_{\delta}
\end{aligned}
$$

so that $L_{4}=L_{3}$. Hence the achievable load responses are the same after the disturbance response decoupling step.

We can also check this same fact on the achievable load performance by directly using the matrix formulation in Theorem 2. The original achievable load responses are $P_{11,12}-P_{12} Q P_{21,2}$ with $Q \in \mathbb{R}^{1} \mathbb{\infty}_{\infty}^{1 \times 3}$, and after disturbance response decoupling the achievable load responses are $P_{11,12}-P_{12} Q_{1} U_{2} P_{21,2}$ with $Q_{1} \in \mathbb{R} \mathbb{H}_{\infty}^{1 \times 2}$. We can check that $P_{12}$ is strictly proper and $P_{21,2}$ is proper. Hence we can set $A=\left\{P_{12} Q P_{21,2}\right\}$ and $B=\left\{P_{12} Q_{1} \widetilde{U}_{2} P_{21,2}\right\}$ in Theorem 2 and assign $G_{1}=G_{2}=P_{12}, H_{1}=P_{21,2}$ and $H_{2}=U_{2} P_{21,2}$, so that $I=I$ and $K$ is found as

$$
K=\frac{m_{s} s^{2}+c_{s} s+k_{s}}{d(s)}\left[\begin{array}{cc}
m_{u} s^{2}+c_{s} s+k_{s}+k_{t} & 0 \\
0 & m_{u} s^{2}+k_{t} \\
c_{s} s+k_{s} & 0
\end{array}\right]
$$

Accordingly, the achievable load responses remain the same after disturbance response decoupling (Theorem 2(ii)).

\subsection{The half-car model}

In this section, we shall apply the controller parametrization method to the half-car model shown in figure 4. As in the quarter-car model, the actuators $A_{1}$ and $A_{2}$ are modelled so that the relative displacement across each is equal to a low-pass filtered version of the actuator's command signal, i.e.

$$
\begin{aligned}
& \hat{z}_{s}+l_{1} \hat{z}_{\psi}-\hat{z}_{a_{1}}=\gamma(s) \hat{u}_{1} \\
& \hat{z}_{s}-l_{2} \hat{z}_{\psi}-\hat{z}_{a_{2}}=\gamma(s) \hat{u}_{2}
\end{aligned}
$$

where $\gamma(s)$ is defined as in (8). The linearized dynamic equations can be expressed as

$$
\begin{aligned}
m_{s} \ddot{z}_{s} & =F_{s}-u_{p_{1}}-u_{p_{2}} \\
I_{\psi} \ddot{z}_{\psi} & =F_{\psi}-u_{p_{1}} l_{1}+u_{p_{2}} l_{2} \\
m_{1} \ddot{z}_{u_{1}} & =u_{p_{1}}+F_{r_{1}} \\
m_{2} \ddot{z}_{u_{2}} & =u_{p_{2}}+F_{r_{2}}
\end{aligned}
$$

where the passive suspension forces $u_{p_{1}}, u_{p_{2}}$, and the tyre forces $F_{r_{1}}, F_{r_{2}}$ are given by

$$
\begin{aligned}
u_{p_{1}} & =c_{1}\left(\dot{z}_{s}+l_{1} \dot{z}_{\psi}-\dot{z}_{u_{1}}\right)+k_{1}\left(z_{a_{1}}-z_{u_{1}}\right) \\
u_{p_{2}} & =c_{2}\left(\dot{z}_{s}-l_{2} \dot{z}_{\psi}-\dot{z}_{u_{2}}\right)+k_{2}\left(z_{a_{2}}-z_{u_{2}}\right) \\
F_{r_{1}} & =k_{t_{1}}\left(z_{r_{1}}-z_{u_{1}}\right) \\
F_{r_{2}} & =k_{t_{2}}\left(z_{r_{2}}-z_{u_{2}}\right)
\end{aligned}
$$

We now write the system in the form of figure 1 with $z_{1}=\left[z_{s}, z_{\psi}, z_{u_{1}}, z_{u_{2}}\right]^{\prime}, \quad w_{1}=\left[z_{r_{1}}, z_{r_{2}}\right]^{\prime}, \quad w_{2}=\left[F_{s}, F_{\psi}\right]^{\prime}$,

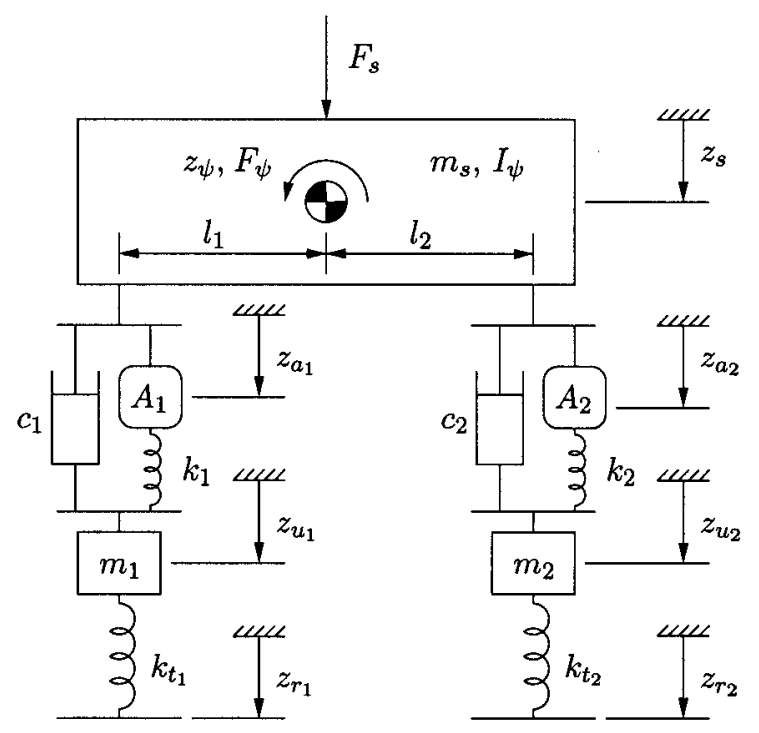

Figure 4. The half-car model. 
$y=\left[\ddot{z}_{s}, \ddot{z}_{\psi}, D_{1}, D_{2}\right]^{\prime}$ where $D_{1}=z_{s}+l_{1} z_{\psi}-z_{u 1}, D_{2}=$ $z_{s}-l_{2} z_{\psi}-z_{u 2}$ are strut deflections, $u=\left[u_{1}, u_{2}\right]^{\prime}$ as in (20), (21) and $z_{2}$ omitted. It can be checked that $p_{3}=4$, $r_{2}=r_{3}=2$. The matrix $\widetilde{U}_{2} \in \mathbb{R} \mathbb{H}_{\infty}^{\left(p_{3}-r_{3}\right) \times p_{3}}$ given by

$$
\tilde{U}_{2}=\left[\begin{array}{cccc}
\frac{m_{s} l_{2}}{\left(c_{1} s+k_{1}\right)\left(l_{1}+l_{2}\right)} & \frac{I_{\psi}}{\left(c_{1} s+k_{1}\right)\left(l_{1}+l_{2}\right)} & 1 & 0 \\
\frac{m_{s} l_{1}}{\left(c_{2} s+k_{2}\right)\left(l_{1}+l_{2}\right)} & \frac{-I_{\psi}}{\left(c_{2} s+k_{2}\right)\left(l_{1}+l_{2}\right)} & 0 & 1
\end{array}\right]
$$

satisfies $\tilde{U}_{2} P_{21,1}=0$. Thus the conditions of Theorem 1(iii) hold.

Now we discuss the achievable load responses of the half-car model after disturbance response decoupling. All stabilizing controllers a reparametrized by (2) and the load responses are $P_{11,12}-P_{12} Q P_{21,2}$ with $Q \in \mathbb{R}^{2} \mathbb{H}_{\infty}^{2 \times 4}$. After the disturbance response decoupling procedure, the load responses become $P_{11,12}-$ $P_{12} Q_{1} U_{2} P_{21,2}$, where $U_{2}$ is given by (26) and $Q_{1} \in \mathbb{R} \mathbb{H}_{\infty}^{2 \times 2}$. Therefore, we consider two sets of strictly proper $\sim$ matrices: $A=\left\{P_{12} Q P_{21,2}\right\}$ and $B=$ $\left\{P_{12} Q_{1} \widetilde{U}_{2} P_{21,2}\right\}$ in Theorem $2_{2}$ which gives $G_{1}=$ $G_{2}=P_{12}, H_{1}=P_{21,2}$ and $H_{2}=U_{2} P_{21,2}$. We find $I=I$ and $K$ as

$$
\boldsymbol{K}=H_{1} H_{2}^{-1}=\frac{1}{p_{8}(s)}\left[\begin{array}{ll}
o\left(s^{9}\right) & o\left(s^{9}\right) \\
o\left(s^{9}\right) & o\left(s^{9}\right) \\
o\left(s^{7}\right) & o\left(s^{7}\right) \\
o\left(s^{7}\right) & o\left(s^{7}\right)
\end{array}\right]
$$

where $p_{8}(s)$ is an eighth order Hurwitz polynomial and $o\left(s^{i}\right)$ indicates an $i$ th order polynomial. Thus, from Theorem 2, the achievable load responses after disturbance response decoupling can approximate the original load responses to an error of $\epsilon$ in the $H_{\infty}$ norm.

\section{Numerical example}

\subsection{Calculation of the controller structure}

A key step in the disturbance response decoupling designs is the computation of the matrix $U_{2}$ which determines the required controller structure. Throughout the application of vehicle active suspension design in Smith and Wang (2002), it was always possible to calculate $U_{2}$ symbolically using Maple. However, for more complicated vehicle models this may not be feasible. A basis for a numerical approach, which was found to be tractable in a variety of examples (which all satisfied $r_{3}=m_{1}$ ), used the following sequence of steps

(1) find a factorization $P_{21,1}=U_{1} F$ where $U_{1}, F$ are stable, $F$ is square, and $U_{1}=\left[M^{\prime}, N^{\prime}\right]^{\prime}$ with $M(\infty)$ being square and non-singular;

(2) find a minimal realization of $L:=N M^{-1}$;

(3) find a left coprime factorization $L=\widetilde{M}^{-1} \tilde{N}$ and set $U_{2}=[-N, M]$ (e.g., see Zhou et al. 1996, Theorem 12.19)).

In our examples it was always possible to carry out (1) in an ad hoc manner by considering the relative degrees of the elements of $P_{21,1}$. Indeed, in the example below it was possible to choose $F=I$. We note the connection with Theorem 1 that $U_{2}$ constructed in (3) is a left annihilator of $P_{21,1}$ and is a part of a unimodular matrix.

\subsection{Double-wishbone model}

We now consider the half-car double-wishbone model, as shown in figure 5. The left (right) suspension strut consists of a passive damper of constant $c_{1}\left(c_{2}\right)$ in parallel with a series combination of actuators $A_{1}\left(A_{2}\right)$ and a spring of constant $k_{1}\left(k_{2}\right)$ (this is a 'Sharp' actuator as introduced in $\S 3$ ). The actuators are modelled in such a way that the relative displacement across each is equal to a low-pass filtered version of the actuator's

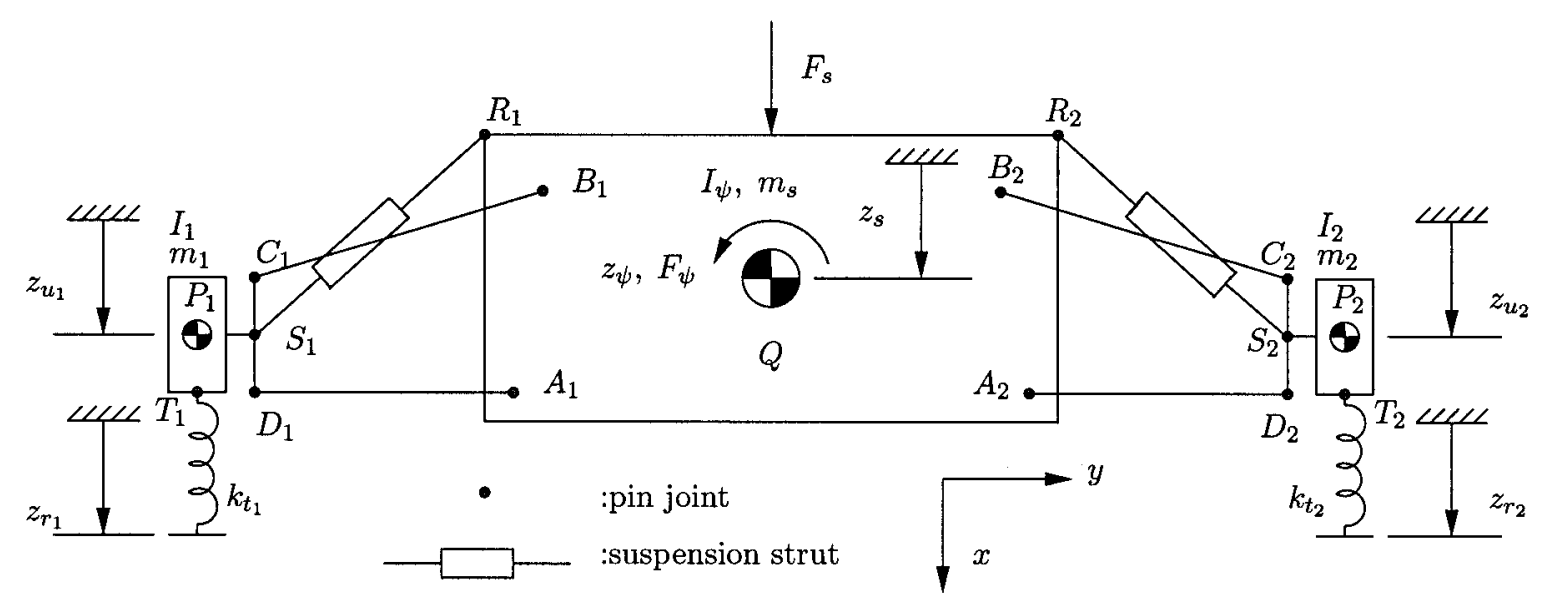

Figure 5. The half-car double-wishbone model. 

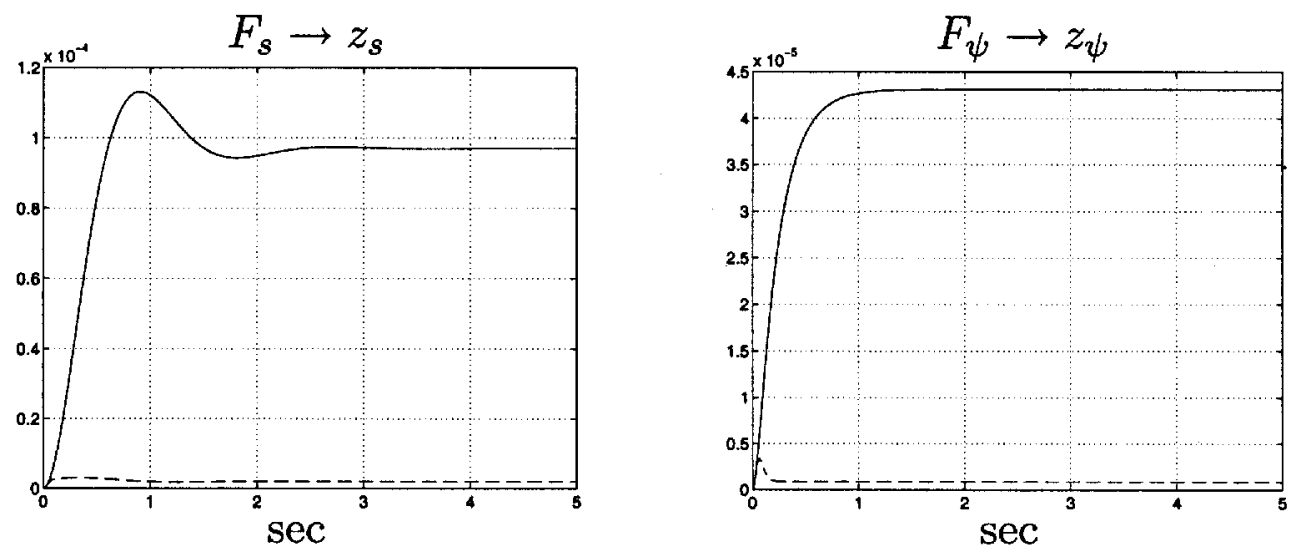

Figure 6. Step responses for linearlized model of $T_{\hat{F}_{s} \rightarrow \hat{z}_{s}}$ and $T_{\hat{F}_{\psi} \rightarrow \hat{z}_{\psi}}$ : passive (solid) and active control (dashed)

command signal, as in (20) and (21). The tyre forces are modelled as being always vertical. This model was built and linearized by AutoSim. We will analyse the linearized model and find the controller structure which improves the load responses and keeps the road responses the same as in the passive case.

We now write the system in the form of figure 1 with $z_{1}=\left[z_{s}, z_{\psi}, z_{u_{1}}, z_{u_{2}}\right]^{\prime}, \quad w_{1}=\left[z_{r_{1}}, z_{r_{2}}\right]^{\prime}, \quad w_{2}=\left[F_{s}, F_{\psi}\right]$, $y=\left[\ddot{z}_{s}, \ddot{z}_{\psi}, D_{1}, D_{2}\right]^{\prime}$ where $D_{1}$ and $D_{2}$ are the strut deflections and $z_{2}$ omitted. The following parameters will be used for the model: $m_{s}=625 \mathrm{~kg}$, $I_{\psi}=170 \mathrm{~kg} \mathrm{~m}^{2}, \quad m_{1}=m_{2}=50 \mathrm{~kg}, \quad I_{1}=I_{2}=0.1 \mathrm{~kg} \mathrm{~m}^{2}$, $c_{1}=c_{2}=1 \mathrm{kNs} / \mathrm{m}, \quad k_{1}=k_{2}=4 \mathrm{kN} / \mathrm{m}, \quad k_{t_{1}}=k_{t_{2}}=$ $250 \mathrm{kN} / \mathrm{m}$. Those parameters are chosen to give satisfactory (i.e. soft) road responses, so that we can apply the disturbance response decoupling design for the load responses. The actuator dynamics are modelled as in (8) with $\omega_{n}=100 \mathrm{rad} / \mathrm{sec}$ and $\delta=0.707$. The geometric layout of the model in the nominal configuration is given by the following coordinates

$$
\begin{aligned}
Q & =(0,0) & & A_{1}=(0.25,-1) \\
B_{1} & =(-0.05,-0.94) & & C_{1}=(0.15,-1.4) \\
D_{1} & =(0.25,-1.4) & & R_{1}=(-0.25,-1.04) \\
S_{1} & =(0.2,-1.4) & & P_{1}=(0.2,-1.5) \\
T_{1} & =(0.35,-1.5) & & A_{2}=(0.25,1) \\
B_{2} & =(-0.05,0.94) & & C_{2}=(0.15,1.4) \\
D_{2} & =(0.25,1.4) & & R_{2}=(-0.25,1.04) \\
S_{2} & =(0.2,1.4) & & P_{2}=(0.2,1.5) \\
P_{2} & =(0.35,1.5) & &
\end{aligned}
$$

in units of meters. We find the $\widetilde{U}_{2}$ matrix, after model reduction (using balanced truncation from 8 th to 5 th order), to be as shown in equation (28) below. (The $L_{\infty}$-norm error in $U_{2}$ due to model reduction was $4.1243 \times 10^{-7}$. The $H_{\infty}$ norm of $\widetilde{U}_{2} P_{21,1}$ was equal to $5.73 \times 10^{-14}$ and $5.42 \times 10^{-12}$ before and after model reduction, so the reduced order $U_{2}$ was considered as still preserving disturbance response decoupling property.) Using the $H_{\infty}$ loop shaping method (Zhou et al. 1996) with the weighting function

$$
W_{1}=\frac{8(s+80)}{s+2}\left[\begin{array}{ll}
1 & 0 \\
0 & 1
\end{array}\right]
$$

we obtain a $K_{1}$ (after model reduction using balanced truncation from 12 th to 5 th order) as

$$
K_{1}=\left[\begin{array}{ll}
K_{1,11} & K_{1,12} \\
K_{1,21} & K_{1,22}
\end{array}\right]
$$

where $K_{1,12}=K_{1,21}=0$ and

$K_{1,11}=K_{1,22}=$

$\frac{-26.05(s+21.80)(s+54.48)(s+114.34)(s+70.11 \pm 69.98 j)}{(s+2)(s+55.22)(s+165.48)(s+66.23 \pm 120.51 j)}$ $(s+2)(s+55.22)(s+165.48)(s+66.23 \pm 120.51 j)$

The controller gives desirable load responses, as shown in figure 6. In addition it reduces $T_{\hat{F}_{s} \rightarrow \hat{z}_{s}}(0)$ from $9.70 \times 10^{-5}$ to $1.95 \times 10^{-6}$ and $T_{\hat{F}_{w} \rightarrow \hat{z}_{w}}(0)$ from $4.31 \times 10^{-5}$ to $8.65 \times 10^{-7}$, when compared to the passive case (i.e. no feedback). The simulation results show that the road disturbance responses are the same as the open loop.

$$
\tilde{U}_{2}=\left[\begin{array}{cccc}
\frac{26.79}{(s+4.20)(s+93.65)} & \frac{5.96}{(s+4.14)(s+118.79)} & \frac{-(s+4.16)}{s+4.17} & \frac{9.4 \times 10^{-3}}{s+4.17} \\
\frac{26.79}{(s+4.20)(s+93.65)} & \frac{-5.96}{(s+4.14)(s+118.79)} & \frac{9.4 \times 10^{-3}}{s+4.17} & \frac{-(s+4.16)}{s+4.17}
\end{array}\right]
$$




\section{Concluding remarks}

This paper has presented two new results on disturbance response decoupling. The first (Theorem 1) established the required control structure in terms of a left annihilator of a plant open-loop transfer function. The second (Theorem 2) gave conditions to determine if the achievable performance for other disturbance paths would be reduced by the parametrization. We remark that Theorem 2 can also be applied to study achievable performance for the general case of Smith and Wang (2002).

The approach was illustrated for standard quarterand half-car models with various different choices of measurements. The required control structures were derived in parametric form. A controller structure was calculated numerically for a half-car double-wishbone model.

\section{References}

Hrovat, D., and Hubbard, M., 1981, Optimal vehicle suspensions minimizing rms rattlespace, sprung-mass acceleration and jerk Transactions of the ASME, 103, 228-236.

Pitcher, R., Hillel, H., and Curtis, C. H., 1977, Hydraulic suspensions with particular reference to public service vehicles Public Service Vehicles Conference (London: Mechanical Engineering Publications).

Shar P, R. S., and Hassan, S. A., 1986, The relative performance capabilities of passive, active and semi-active car suspension systems. Proceedings of the Institution of Mechanical Engineers, 200, 219-228.
Sharp, R. S., and Hassan, S. A., 1986, On the performance capabilities of active automobile suspension systems of limited bandwidth. Vehicle System Dynamics, 16, 213-225.

Smith, M. C., 1995, Achievable dynamic response for automotive active suspension. Vehicle System Dynamics, 24, 133.

Smith, M. C., and Wang, F.-C., 2002, Controller parametrisation for disturbance response decoupling: Application to vehicle active suspension control. IEEE Transactions on Control Systems Technology, 10, 393-407.

Thompson, A. G., 1971, Design of active suspensions. Proceedings of the Institution of Mechanical Engineers 185, 553-563.

VIDYASaGaR, M., 1985, Control System Synthesis: A Factorisation Approach (Cambridge, MA: MIT Press).

Wang, F.-C., and Smith, M. C., 2001, Active and passive suspension control for vehicle dive and squat. Automotive Control Workshop, Lund, Sweden. In R. Johansson and A. Rantzer (eds) Nonlinear and Hybrid Systems in Automotive Control (London: Springer-Verlag).

Williams, R. A., and Best, A., 1994, Control of a low frequency active suspension. IEE Control '94, Conference Publication, 1, 338-343.

Williams, R. A., Best, A., and Crawford, I. L., 1993, Refined low frequency active suspension. International Conference on Vehicle Ride and Handling, Proceedings of the ImechE, C466/0281, 285-300.

Wright, P. G., and Williams, D. A., 1984, The application of active suspension to high performance road vehicles. Proceedings of IMecE Conference on Microprocessors in Fluid Power Engineering (London: Mechanical Engineering Publications), C239/84, 23-28.

Wright, P. G., and Williams, D. A., 1989, The case for an irreversible active suspension system. SAE Transactions, Journal of Passenger Cars, Section 6, pp. 83-90.

Zhou, K., Doyle, J. C., and Glover, K., 1996, Robust and Optimal Control (Upper Saddle River, NJ: Prentice-Hall). 
Copyright of International Journal of Control is the property of Taylor \& Francis Ltd and its content may not be copied or emailed to multiple sites or posted to a listserv without the copyright holder's express written permission. However, users may print, download, or email articles for individual use. 